\title{
New single- and double-parton scattering mechanisms for double charmed meson production
}

\author{
Antoni Szczurek* \\ Institute of Nuclear Physics, Polish Academy of Sciences, Radzikowskiego 152, \\ PL-31-342 Kraków, Poland \\ E-mail: antoni.szczurek@ifj.edu.pl \\ Rafał Maciuła \\ Institute of Nuclear Physics, Polish Academy of Sciences, Radzikowskiego 152, \\ PL-31-342 Kraków, Poland \\ E-mail: rafal.maciula@ifj.edu.pl
}

Vladimir A. Saleev

Samara National Research University, Moscow Highway, 34, 443086, Samara, Russia

E-mail: saleev@samsu.ru

\section{Alexandra V. Shipilova}

Samara National Research University, Moscow Highway, 34, 443086, Samara, Russia

E-mail: alexshipilova@samsu.ru

\begin{abstract}
We discuss charm meson-meson pair production recently observed by the LHCb Collaboration at $\sqrt{s}=7 \mathrm{TeV}$ in proton-proton scattering. We examine double-parton scattering (DPS) mechanisms of double $c \bar{c}$ production and following $c c \rightarrow D^{0} D^{0}$ hadronization as well as double $g$ and mixed $g c \bar{c}$ production with $g g \rightarrow D^{0} D^{0}$ and $g c \rightarrow D^{0} D^{0}$ hadronization calculated with the help of the scale-dependent KKKS08 fragmentation functions. A new single-parton scattering (SPS) mechanism of $g g$ production is also taken into consideration. Calculated differential distributions as a function of transverse momentum $p_{T}$ of one of the $D^{0}$ mesons, pair invariant mass $M_{D^{0} D^{0}}$ and azimuthal angle $\varphi_{D^{0} D^{0}}$ distributions are confronted with the measured ones. The manifestation of the new SPS mechanisms with $g \rightarrow D^{0}$ fragmentation within the scale-dependent fragmentation scheme change the overall picture suitable for standard scale-independent fragmentation where only DPS $c c \rightarrow D^{0} D^{0}$ mechanism is present. Some consequences of the new mechanisms are discussed.
\end{abstract}

XXIV International Workshop on Deep-Inelastic Scattering and Related Subjects 11-15 April, 2016

DESY Hamburg, Germany

\footnotetext{
*Speaker.

${ }^{\dagger}$ The work has been supported by the Polish National Science Center grant DEC-2014/15/B/ST2/02528.
} 


\section{Introduction}

At present, double charm production is expected to be one of the most promising channels for studies of double-parton scattering (DPS) effects at the LHC. This was predicted [1] and further supported by the experimental observations reported by the LHCb Collaboration [2]. Next, the phenomenology of $D D$ meson-meson pair production was carefully examined in the $k_{t}$-factorization approach and a relatively good description of the LHCb experimental data was achieved for both the total yield and the dimeson correlation observables [3]. In the theoretical analyses, both, single- and double-parton scattering mechanisms were taken into consideration. The contribution of single-parton scattering (SPS) mechanism $g g \rightarrow c \bar{c} c \bar{c}$, discussed in detail in the collinear [4] and $k_{t}$-factorization [5] approaches, was found to be rather small and definitely not able to describe relatively large $D D$ cross sections measured by the LHCb.

The phenomenological studies of the $D D$ pair production were based on the rather standard fragmentation scheme with scale-independent Peterson fragmentation function (FF) [6], where only $c \rightarrow D$ transition is included. However, an alternative approach is to apply scale-dependent FFs that undergo DGLAP evolution equations, e.g. KKKS08 model [7], where each parton (gluon, $u, d, s, \bar{u}, \bar{d}, \bar{s}, c, \bar{c})$ can contribute to $D$ meson production. In the latter scenario, the $c \rightarrow D$ contribution is reduced by the evolution of the FF but a very important contribution from $g \rightarrow D$ fragmentation appears (see e.g. Ref. [8]).

In this presentation we report on first investigation how important is the gluon fragmentation mechanism for the double $D$-meson production.

\section{A sketch of the theoretical formalism}
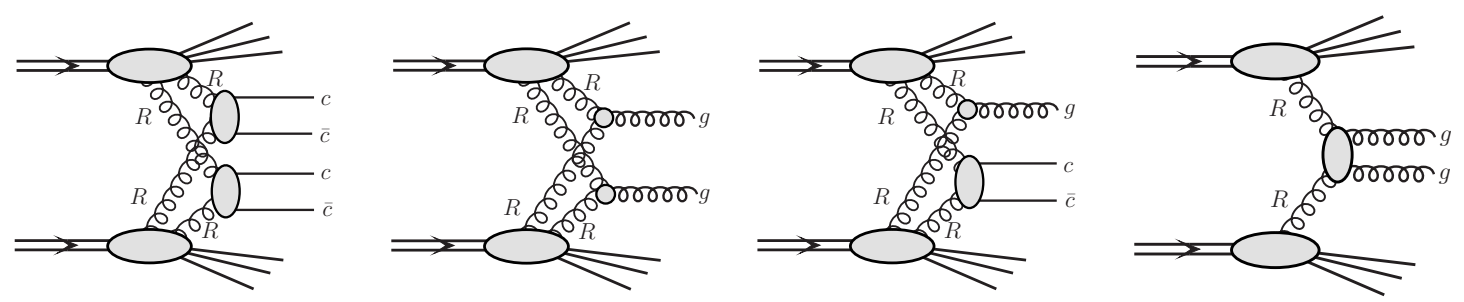

Figure 1: A diagrammatic illustration of the considered mechanisms.

We will compare numerical results for double $D$-meson production obtained with the two different fragmentation schemes. In the (new) scenario with scale-dependent KKKS08 FFs and $g \rightarrow D$ fragmentation the number of contributing processes grows compared to the standard (old) scenario with $c \rightarrow D$ fragmentation only. According to the new scenario one has to consider more processes for single $D$ meson production ( $c$ and $g \rightarrow D$ components). This also causes an extension of the standard DPS $D D$ pair production by new mechanisms. In addition to the coventional DPS $c c \rightarrow D D$ (left diagram in Fig.1) considered in Refs. [3, 4, 5] there is a double $g \rightarrow D$ (or double $g \rightarrow \bar{D}$ ) fragmentation mechanism, called here DPS $g g \rightarrow D D$ (middle-left diagram in Fig.1) as well as the mixed DPS $g c \rightarrow D D$ contribution (middle-right diagram in Fig.1). 
As a consequence of the new approach a new SPS $g g \rightarrow D D$ mechanism shows up (right diagram in Fig.1). In this case the two produced gluons are correlated in azimuth and the mechanism will naturally lead to an azimuthal correlation between the two $D$ mesons. Such a correlation was actually observed in the LHCb experimental data [2] and could not be explained by the SPS $2 \rightarrow 4$ perturbative $g g \rightarrow c \bar{c} c \bar{c}$ contribution (see e.g. Ref. [5]) which is very small.

DPS cross section for production of $c c, g g$ or $g c$ system, assuming factorization of the DPS model, can be written as:

$$
\begin{aligned}
& \frac{d \sigma^{D P S}(p p \rightarrow c c X)}{d y_{1} d y_{2} d^{2} p_{1, t} d^{2} p_{2, t}}=\frac{1}{2 \sigma_{e f f}} \cdot \frac{d \sigma^{S P S}\left(p p \rightarrow c \bar{c} X_{1}\right)}{d y_{1} d^{2} p_{1, t}} \cdot \frac{d \sigma^{S P S}\left(p p \rightarrow c \bar{c} X_{2}\right)}{d y_{2} d^{2} p_{2, t}} \\
& \frac{d \sigma^{D P S}(p p \rightarrow g g X)}{d y_{1} d y_{2} d^{2} p_{1, t} d^{2} p_{2, t}}=\frac{1}{2 \sigma_{e f f}} \cdot \frac{d \sigma^{S P S}\left(p p \rightarrow g X_{1}\right)}{d y_{1} d^{2} p_{1, t}} \cdot \frac{d \sigma^{S P S}\left(p p \rightarrow g X_{2}\right)}{d y_{2} d^{2} p_{2, t}} \\
& \frac{d \sigma^{D P S}(p p \rightarrow g c X)}{d y_{1} d y_{2} d^{2} p_{1, t} d^{2} p_{2, t}}=\frac{1}{\sigma_{e f f}} \cdot \frac{d \sigma^{S P S}\left(p p \rightarrow g X_{1}\right)}{d y_{1} d^{2} p_{1, t}} \cdot \frac{d \sigma^{S P S}\left(p p \rightarrow c \bar{c} X_{2}\right)}{d y_{2} d^{2} p_{2, t}}
\end{aligned}
$$

The often called pocket-formula is a priori a severe approximation. The flavour, spin and color correlations lead, in principle, to interference effects that result in its violation as discussed e.g. in Ref. [9]. Even for unpolarized proton beams, the spin polarization of the two partons from one hadron can be mutually correlated, especially when the partons are relatively close in phase space (having comparable $x$ 's). Moreover, in contrast to the standard single PDFs, the two-parton distributions have a nontrivial color structure which also may lead to a non-negligible correlations effects. Such effects are usually not included in phenomenological analyses. They were exceptionally discussed in the context of double charm production [10]. However, the effect on e.g. azimuthal correlations between charmed quarks was found there to be very small, much smaller than effects of the SPS contribution associated with double gluon fragmentation discussed here. In addition, including perturbative parton splitting mechanism also leads to a breaking of the pocket-formula [11]. This formalism was so far formulated for the collinear leading-order approach which for charm (double charm) may be a bit academic as this leads to underestimation of the cross section. Imposing sum rules also leads to a breaking of the factorized Ansatz but the effect almost vanishes for small longitudinal momentum fractions [12]. Taken the above we will use the pocket-formula in the following.

All the considered mechanisms (see Fig. 1) are calculated in the $k_{t}$-factorization approach with off-shell initial state partons and unintegrated ( $k_{t}$-dependent) PDFs (unPDFs). Fully gauge invariant treatment of the initial-state off-shell gluons and quarks can be achieved in the $k_{t}$-factorization approach only when they are considered as Reggeized gluons or Reggeons. The relevant Reggeized amplitudes can be presented using Fadin-Kuraev-Lipatov effective vertices. The useful analytical formulae for $\overline{\left|\mathscr{M}_{R R \rightarrow g}\right|^{2}}, \overline{\left|\mathscr{M}_{R R \rightarrow g g}\right|^{2}}$ and $\overline{\left|\mathscr{M}_{R R \rightarrow c \bar{c}}\right|^{2}}$ squared amplitudes used in the calculations here can be found in Refs. [8, 13]. We use the LO Kimber-Martin-Ryskin (KMR) unPDFs, generated from the LO set of a up-to-date MMHT2014 collinear PDFs fitted also to the LHC data. In the perturbative part of the calculations we use a running LO $\alpha_{S}$ provided with the MMHT2014 PDFs. The charm quark mass is set to $m_{c}=1.5 \mathrm{GeV}$ and the renormalization and factorization scales are 
equal to $\mu^{2}=p_{t}^{2}$ for $R R \rightarrow g$ subprocess, to the averaged transverse momentum $\mu^{2}=\left(p_{1 t}^{2}+p_{2 t}^{2}\right) / 2$ for $R R \rightarrow g g$, and to the averaged transverse mass $\mu^{2}=\left(m_{1 t}^{2}+m_{2 t}^{2}\right) / 2$ for $R R \rightarrow c \bar{c}$ case, where $m_{t}=\sqrt{p_{t}^{2}+m_{c}^{2}}$ (for more details see Ref.[14]).

In order to calculate correlation observables for two mesons we follow here, similar as in the single meson case, the fragmentation function technique for hadronization process:

$$
\begin{aligned}
\frac{d \sigma^{D P S}(p p \rightarrow D D X)}{d y_{1} d y_{2} d^{2} p_{1 t}^{D} d^{2} p_{2 t}^{D}} & =\int \frac{D_{c \rightarrow D}\left(z_{1}, \mu\right)}{z_{1}} \cdot \frac{D_{c \rightarrow D}\left(z_{2}, \mu\right)}{z_{2}} \cdot \frac{d \sigma^{D P S}(p p \rightarrow c c X)}{d y_{1} d y_{2} d^{2} p_{1 t}^{c} d^{2} p_{2 t}^{c}} d z_{1} d z_{2} \\
& +\int \frac{D_{g \rightarrow D}\left(z_{1}, \mu\right)}{z_{1}} \cdot \frac{D_{g \rightarrow D}\left(z_{2}, \mu\right)}{z_{2}} \cdot \frac{d \sigma^{D P S}(p p \rightarrow g g X)}{d y_{1} d y_{2} d^{2} p_{1 t}^{g} d^{2} p_{2 t}^{g}} d z_{1} d z_{2} \\
& +\int \frac{D_{g \rightarrow D}\left(z_{1}, \mu\right)}{z_{1}} \cdot \frac{D_{c \rightarrow D}\left(z_{2}, \mu\right)}{z_{2}} \cdot \frac{d \sigma^{D P S}(p p \rightarrow g c X)}{d y_{1} d y_{2} d^{2} p_{1 t}^{g} d^{2} p_{2 t}^{c}} d z_{1} d z_{2}
\end{aligned}
$$

where: $p_{1 t}^{g, c}=\frac{p_{1, t}^{D}}{z_{1}}, p_{2, t}^{g, c}=\frac{p_{2 t}^{D}}{z_{2}}$ and meson momentum fractions $z_{1}, z_{2} \in(0,1)$.

The same formula for SPS $D D$-production via fragmentation of each of the gluon reads

$$
\frac{d \sigma_{g g}^{S P S}(p p \rightarrow D D X)}{d y_{1} d y_{2} d^{2} p_{1 t}^{D} d^{2} p_{2 t}^{D}} \approx \int \frac{D_{g \rightarrow D}\left(z_{1}, \mu\right)}{z_{1}} \cdot \frac{D_{g \rightarrow D}\left(z_{2}, \mu\right)}{z_{2}} \cdot \frac{d \sigma^{S P S}(p p \rightarrow g g X)}{d y_{1} d y_{2} d^{2} p_{1 t}^{g} d^{2} p_{2 t}^{g}} d z_{1} d z_{2},
$$

where: $p_{1 t}^{g}=\frac{p_{1, t}^{D}}{z_{1}}, p_{2, t}^{g}=\frac{p_{2 t}^{D}}{z_{2}}$ and meson momentum fractions $z_{1}, z_{2} \in(0,1)$.

\section{Comparison to the $\mathrm{LHCb}$ data}

Before we start a comparison of the theoretical results with the LHCb double charm data we wish to stress that the both fragmentation schemes considered here lead to a very good (and very similar) description of the LHCb data for inclusive single $D$ meson production [14]. So both prescriptions together with the $k_{t}$-factorization approach seem to be a good and legitimate starting points for double charm production studies.
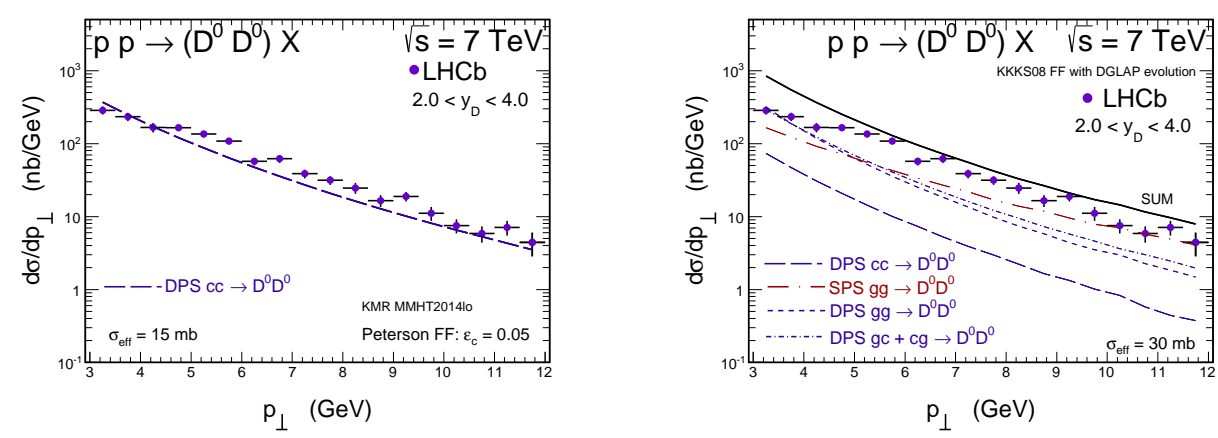

Figure 2: $D^{0}$ meson transverse momentum distribution within the LHCb acceptance region. The left panel is for the first scenario and for Peterson $c \rightarrow D$ fragmentation function while the right panel is for the second scenario and for the fragmentation function that undergo DGLAP evolution equation. 
Now we wish to compare results of our theoretical approach for double charm production described briefly in the previous section with the $\mathrm{LHCb}$ experimental data for $D^{0} D^{0}$ pair production. In Fig. 2 we compare results of our calculation with experimental distribution in transverse momentum of one of the meson from the $D^{0} D^{0}$ pair. We show results for the first scenario when standard Peterson FF is used for the $c \rightarrow D^{0}$ fragmentation (left panel) as well as the result for the second scenario when the KKKS08 FFs with DGLAP evolution for $c \rightarrow D^{0}$ and $g \rightarrow D^{0}$ are used. One can observe that the DPS $c c \rightarrow D^{0} D^{0}$ contribution in the new scenario is much smaller than in the old scenario. In addition, the slope of the distribution in transverse momentum changes. Both the effects are due to evolution of corresponding FF in the second scenario, compared to lack of such an effect in the first scenario. The different new mechanisms shown in Fig. 1 give contributions of similar size. We can obtain a better agreement in the second case provided $\sigma_{\text {eff }}$ parameter is increased from $15 \mathrm{mb}$ to $30 \mathrm{mb}$. Even then we overestimate the $\mathrm{LHCb}$ data for $3<p_{T}<5 \mathrm{GeV}$.
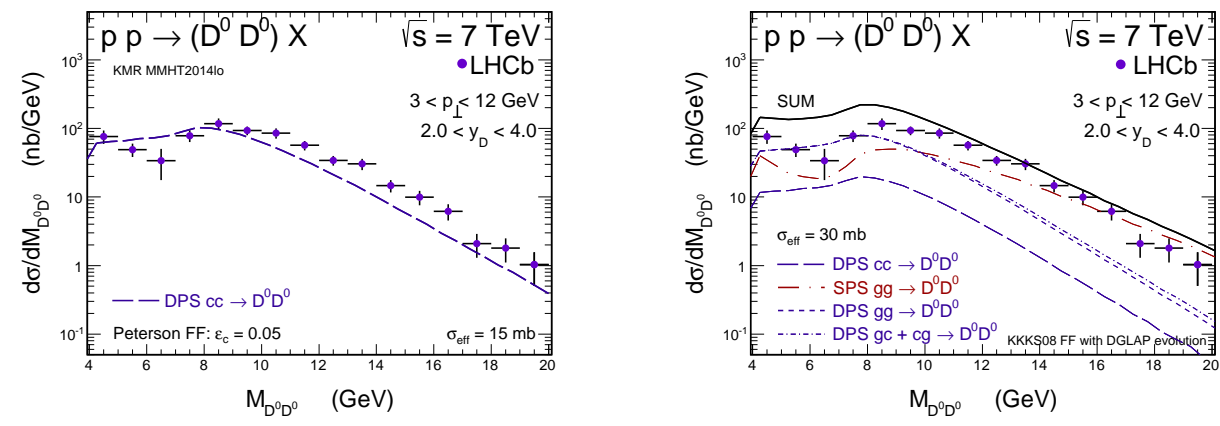

Figure 3: The same as in the previous figure but for $M_{D^{0} D^{0}}$ dimeson invariant mass distribution.
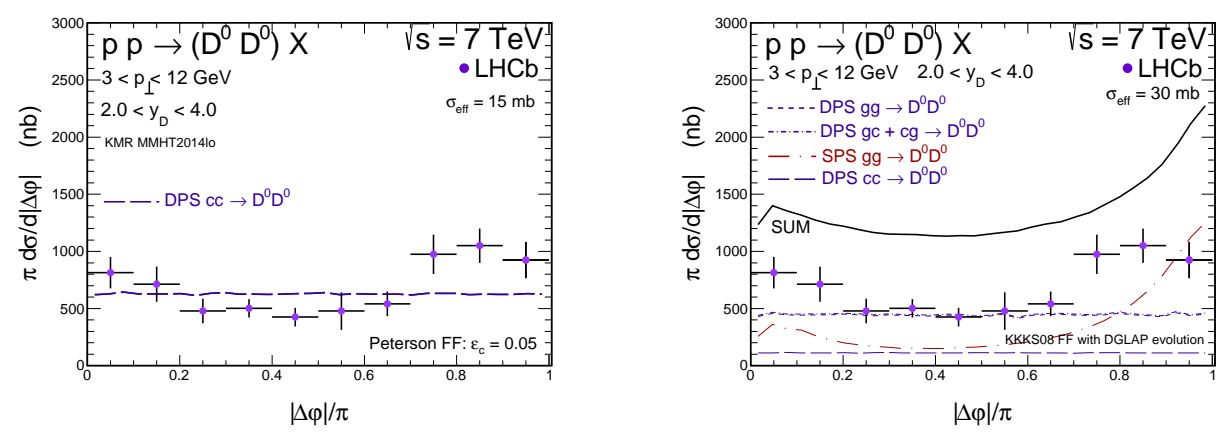

Figure 4: The same as in the previous figure but for the distribution in azimuthal angle $\varphi_{D^{0} D^{0}}$.

In Fig. 3 we show dimeson invariant mass distribution $M_{D^{0} D^{0}}$ again for the two cases considered. In the first scenario we get a good agreement only for small invariant masses while in the second scenario we get a good agreement only for large invariant masses. The large invariant masses are strongly correlated with large transverse momenta, so the situation here (for the invariant mass distribution) is quite similar as in Fig. 2 for the transverse momentum distribution.

In Fig. 4 we show azimuthal angle correlation $\varphi_{D^{0} D^{0}}$ between $D^{0}$ and $D^{0}$. While the correlation function in the first scenario is completely flat, the correlation function in the second scenario shows 
some tendency similar as in the experimental data. The observed overestimation comes from the region of small transverse momenta.

\section{Conclusions}

In the present paper we have discussed production of $D^{0} D^{0}$ pairs in proton-proton collisions at the LHC. We have considered the DPS mechanism of double $c \bar{c}$ production and subsequent double hadronization of two $c$ quarks or two $\bar{c}$ antiquarks using $c \rightarrow D^{0}$ or $c \rightarrow \bar{D}^{0}$ FFs that undergo DGLAP evolution. Furthermore, we have included also production of $g g$ (both SPS and DPS) and DPS $g c$ final states and their subsequent hadronization to the neutral pseudoscalar $D$ mesons.

When added together the new mechanisms with adjusted $\sigma_{\text {eff }}$ give similar result as in the first scenario with one subprocess $(c c \rightarrow D D)$ and scale-independent FF. However, some correlation observables, such as dimeson invariant mass or azimuthal correlations between $D$ mesons, are slightly better described.

In our calculation, within the second scenario a larger value of $\sigma_{e f f}$ is needed to describe the $\mathrm{LHCb}$ data than found from the review of several experimental studies of different processes. This can be partially understood by a lower contribution of perturbative-parton splitting as found in Ref. [11] and/or due to nonperturbative correlations in the nucleon which may lead to transverse momentum dependent $\sigma_{\text {eff }}$. Clearly more involved studies are needed to understand the situation in detail. Some problem may be also related to the fact that the FFs used in the second scenario were obtained in the DGLAP formalism with massless $c$ quarks and $\bar{c}$ antiquarks which may be a too severe approximation, especially for low factorization scales (i.e. low transverse momenta). We expect that including mass effects in the evolution would lower the $g \rightarrow c$ fragmentation.

The presence of the new SPS mechanism may mean that the extraction of $\sigma_{\text {eff }}$ directly from the LHCb experimental data [2] may be not correct.

For more references and details of the calculations presented here we refer the reader to our regular article [14].

\section{References}

[1] M. Luszczak, R. Maciula and A. Szczurek, Phys. Rev. D 85, 094034 (2012).

[2] R. Aaij et al. [LHCb Collaboration], JHEP 1206, 141 (2012) Addendum: [JHEP 1403, 108 (2014)].

[3] R. Maciula and A. Szczurek, Phys. Rev. D 87, 074039 (2013) [arXiv:1301.4469 [hep-ph]].

[4] A. van Hameren, R. Maciula and A. Szczurek, Phys. Rev. D 89, 094019 (2014).

[5] A. van Hameren, R. Maciula and A. Szczurek, Phys. Lett. B 748, 167 (2015).

[6] C. Peterson, D. Schlatter, I. Schmitt and P. M. Zerwas, Phys. Rev. D 27, 105 (1983).

[7] T. Kneesch, B. A. Kniehl, G. Kramer and I. Schienbein, Nucl. Phys. B 799, 34 (2008).

[8] A. V. Karpishkov, M. A. Nefedov, V. A. Saleev and A. V. Shipilova, Phys. Rev. D 91, 054009 (2015).

[9] M. Diehl, D. Ostermeier and A. Schafer, JHEP 1203, 089 (2012).

[10] M. G. Echevarria, T. Kasemets, P. J. Mulders and C. Pisano, JHEP 1504, 034 (2015).

[11] J. R. Gaunt, R. Maciula and A. Szczurek, Phys. Rev. D 90, 054017 (2014).

[12] K. Golec-Biernat, et al., Phys. Lett. B 750, 559 (2015).

[13] M. A. Nefedov, V. A. Saleev and A. V. Shipilova, Phys. Rev. D 87, 094030 (2013).

[14] R. Maciula, V. A. Saleev, A. V. Shipilova and A. Szczurek, Phys. Lett. B 758, 458 (2016). 